\title{
Heilfasten erhöht die "International Normalized Ratio» bei Gesunden stärker als eine ovo-lacto-vegetarische Diät
}

\author{
Stefan Drinda Françoise Wilhelmi de Toledo \\ Klinik Buchinger Bodensee GmbH, Überlingen, Deutschland
}

\section{Schlüsselwörter \\ Fasten · Koagulation · International Normalized Ratio, INR · Vegetarische Diät}

\section{Zusammenfassung}

Hintergrund: Therapeutisches Fasten hat verschiedene metabolische und endokrine Effekte. Auch eine Wirkung auf die Blutgerinnung ist bekannt. Die Datensammlung untersucht die Wirkung des Fastens nach der Buchinger-Methode im Vergleich zu einer ovo-lacto-vegetarischen Diät von ca. $1800 \mathrm{kcal} /$ Tag auf die INR (International Normalized Ratio) bei Gesunden. Aus den Ergebnissen können auch Rückschlüsse zur Therapiekontrolle von fastenden, antikoagulativ behandelten Patienten gezogen werden. Patienten und Methoden: Fastengruppe: 13 Probanden (9 Frauen, 4 Männer, Durchschnittsalter 25,2 Jahre, BMI individuell jeweils normal $\left(18,5-25,0 \mathrm{~kg} / \mathrm{m}^{2}\right)$, keine relevanten Vorerkrankungen); Diätgruppe: 16 Probanden (14 Frauen, 2 Männer, Durchschnittsalter 24 Jahre, BMI individuell jeweils normal $\left(18,5-25,0 \mathrm{~kg} / \mathrm{m}^{2}\right)$, keine relevanten Vorerkrankungen). Tägliche Bestimmung der INR mit Coagu-Check XS ${ }^{\circledR}$ (Roche Diagnostics, Mannheim, Deutschland) zwischen 10 und 11 Uhr vormittags. Fastenschema: Tag 0: Entlastungstag, 4 Fastentage (Buchinger-Methode); an Tag $140 \mathrm{~g}$ Natriumsulfat (Laxans), Fastenbrechen an Tag 5, ovo-lacto-vegetarischer Aufbau an den Tagen 6 und 7. Kontrollgruppe: ovo-lacto-vegetarische Diät mit ca. $1800 \mathrm{kcal} /$ Tag in drei Mahlzeiten. Ergebnisse: Sowohl durch Fasten als auch durch ovo-lacto-vegetarische Diät kommt es zu zeitabhängigen, signifikanten Veränderungen der INR in beiden Gruppen. Dabei zeigt die INR der Diätgruppe eine fallende und die der Fastengruppe eine steigende Tendenz. Die INR liegt in der Fastengruppe im Vergleich mit der Diätgruppe signifikant höher. Schlussfolgerung: Bei Gesunden sind Veränderungen der INR durch die Interventionen mit Fasten oder einer ovo-lacto-vegetarischen Diät nachweisbar. Fasten führt dabei zu höherer, jedoch noch physiologischer INR. Aus dem Ergebnis kann abgeleitet werden, dass eine engmaschige Gerinnungswertkontrolle zwar nicht bei Gesunden, aber bei auf Cumarinderivate eingestellten Patienten, die mit Heilfasten behandelt werden, notwendig ist.

\author{
Keywords \\ Fasting · Coagulation · International Normalized Ratio, INR · \\ Vegetarian diet
}

\section{Summary}

Fasting Increases the International Normalized Ratio in Healthy Subjects More than an Ovo-Lacto-Vegetarian Diet

Background: Therapeutic fasting leads to various metabolic and hormonal effects, including an effect on coagulation. This controlled study examines the effect of fasting or ovo-lacto-vegetarian diet on the INR (International Normalized Ratio). The findings could improve the monitoring of fasting patients under anticoagulative treatment. Patients und Methods: Fasting Group: 13 healthy subjects (9 women, 4 men, mean age 25.2 years, BMI individually normal $\left(18.5-25.0 \mathrm{~kg} / \mathrm{m}^{2}\right)$; diet group: 16 healthy subjects ( 14 women, 2 men, mean age 24 years, $\mathrm{BMI}$ individually normal $\left(18.5-25.0 \mathrm{~kg} / \mathrm{m}^{2}\right)$. Daily measurements of INR (Coagu-Check XS ${ }^{\circledR}$, Roche Diagnostics, Mannheim, Germany) between 10 and 11 a.m. Fasting schedule: day 0 only fruit, 4 days of fasting (Buchinger method); on the first day: $40 \mathrm{~g}$ sodium sulfate (laxative). Breaking of the fasting on day 5 , ovo-lacto-vegetarian realimentation on days 6 and 7; diet group: ovo-lacto-vegetarian diet $1800 \mathrm{kcal} /$ day (three meals). Results: There are time-dependent, significant changes of INR in both groups. By trend there is an increase of INR in the fasting group and a decrease of INR in the diet group to be found. Fasting leads to a higher INR than an ovo-lacto-vegetarian diet. Conclusion: In healthy subjects, significant INR changes occur during fasting and ovo-lacto-vegetarian diet. In comparison to the diet, fasting leads to significantly higher but still normal INR values. From these results it can be concluded that frequent control of coagulation is negligible in healthy subjects but necessary in patients who take cumarin derivatives and who are following a treatment with therapeutic fasting.

\section{KARGER \\ Fax +497614520714 Information@Karger.d} www.karger.com
Dr. med. Stefan Drinda

Klinik Buchinger Bodense

Wilhelm-Beck-Strasse 27, 88662 Überlingen, Deutschland

Tel. +49 7551-807811, Fax -807189

stefan.drinda@buchinger.com 


\section{Mots-clés}

Jeûne · Coagulation · International Normalized Ratio, INR .

Régime végétarien

\section{Résumé}

Le jeûne thérapeutique induit une augmentation de l'«International Normalized Ratio» plus forte qu'un régime ovo-lacto-végétarien

Contexte: Le jeûne thérapeutique présente différents effets métaboliques et endocriniens. Un effet sur la coagulation sanguine est également connu. Des données ont été collectées afin d'analyser l'effet du jeûne selon la méthode Buchinger par rapport à un régime ovo-lactovégétarien d'env. $1800 \mathrm{kcal} /$ jour sur I'IRN (International Normalized Ratio) chez des sujets sains. Les résultats permettent de tirer des conclusions sur le contrôle thérapeutique de patients jeûneurs suivant un traitement anticoagulant. Patients et méthodes: Groupe jeûne: 13 sujets ( 9 femmes, 4 hommes, âge moyen: 25,2 ans, valeur individuelle de I'IMC toujours normale $\left(18,5-25,0 \mathrm{~kg} / \mathrm{m}^{2}\right)$, aucune maladie préalable pertinente); groupe régime: 16 sujets (14 femmes, 2 hommes, âge moyen: 24 ans, valeur individuelle de I'IMC toujours normale (18,5$25,0 \mathrm{~kg} / \mathrm{m}^{2}$ ), aucune maladie préalable pertinente). Détermination quotidienne de I'IRN au moyen de Coagu-Check XS ${ }^{\circledR}$ (Roche Diagnostics, Mannheim, Allemagne) entre 10 et $11 \mathrm{~h}$ dans la matinée. Schéma du jeûne: jour 0: journée de suppression progressive; 4 jours de jeûne (méthode Buchinger); jour 1: $40 \mathrm{~g}$ de sulfate de sodium (laxatif); jour 5 : interruption du jeûne; jours 6 et 7 reprise du régime ovo-lacto-végétarien. Groupe contrôle: régime ovo-lacto-végétarien avec env. $1800 \mathrm{kcal} /$ jour répartis sur trois repas. Résultats: L'IRN présente dans les deux groupes des modifications significatives et chrono-dépendantes induites aussi bien par le jeûne que par le régime ovo-lacto-végétarien. Le groupe régime montre une tendance à la baisse et le groupe jeûne une tendance à la hausse. L'IRN est significativement plus élevé dans le groupe jeûne que dans le groupe régime. Conclusion: Chez les sujets sains, il est possible de mettre en évidence des modifications de l'IRN induites par le jeûne ou par un régime ovo-lacto-végétarien. Le jeûne entraîne des valeurs d'IRN plus élevées, restant néanmoins physiologiques. Ces résultats permettent de déduire qu'un contrôle étroit des paramètres de coagulation n'est certes pas nécessaire chez les sujets sains, mais l'est chez les patients sous dérivés coumariques et traités par jeûne thérapeutique.

\section{Hintergrund}

Heilfasten wird als komplemetärmedizinische Methode bei unterschiedlichen Erkrankungen eingesetzt [1]. Eine Fastenbehandlung führt zu einer Reihe metabolischer und endokriner Veränderungen, die therapeutisch genutzt werden. Klinisch kontrollierte Studien konnten die Wirksamkeit des Fastens zur Behandlung von Übergewicht [2, 3], entzündlich rheumatischen Erkrankungen [4], aber auch kardiovaskulären Erkrankungen [5] nachweisen. Bereits die Erfahrungswerte zeigen, dass Fasten auch die
Gerinnung beeinflusst [6]. Darüber hinaus wurde die antikoagulative Wirkung des Fastens in verschiedenen Studien [7, 8], auch für normalgewichtige Probanden, nachgewiesen. Das Fasten beeinflusst dabei mehrere Faktoren der Gerinnung - wie den plasmatischen Teil, die Fibrinolyse oder die Thrombozytenaktivierung [7]. Daher werden die Veränderungen der Blutgerinnung während des Fastens nicht allein durch eine reduzierte Vitamin-KZufuhr erklärt, die nachvollziehbar ist und die etwa nach dem 3. Fastentag an Bedeutung gewinnt [9]. Wird das Fasten als Therapie bei Adipositas eingesetzt, werden zusätzliche hormonelle Regelkreise beeinflusst. Eine erhöhte Fettzellmasse verursacht erhöhte PAI-1(PlasminogenAktivator-Inhibitor-1)-Spiegel [10]. Diese führen bei Adipösen $\mathrm{zu}$ einer verminderten Fibrinolyse und somit $\mathrm{zu}$ einer erhöhten Thrombogenität. Ein Fettabbau im Fasten kann die Gerinnungsfähigkeit durch verminderte PAI-1Spiegel «normalisieren»; additiv wirkt auch das Fasten antikoagulativ. Zum Verhalten der Gerinnungswerte von Adipösen bei Fastentherapie sind wenige Datensammlungen [8] publiziert; zu Gesunden und Fastenden sind kontrollierte Studien [7] verfügbar. Hingegen ist zum Verhalten von Gerinnungswerten unter vegetarischer Diät wenig publiziert. Vegetarier haben eine eher verkürzte Blutungszeit und damit eine höhere Koagulationsfähigkeit. Der Umstand wird durch eine höhere Thrombozytenzahl und -aktivität erklärt, die den Effekt verminderter plasmatischer Gerinnungsfaktoren (Fibrinogen, Faktor VIIc, Antithrombin III, Protein S, Plasminogen) zu kompensieren scheinen [11]. Die Frage einer Beeinflussung der plasmatischen Gerinnung durch Fasten ist deshalb relevant, da diese Therapie zur Behandlung des kardiovaskulären Risikofaktors Adipositas genutzt werden kann und ein Teil der fastenden Patienten bereits mit z.B. Cumarinderivaten (z.B. Falithrom ${ }^{\circledR}$, Marcumar $^{\circledR}$, Previscan ${ }^{\circledR}$, Sintrom ${ }^{\circledR}$, Warfarin ${ }^{\circledR}$ ) vorbehandelt ist. Dieser Teil macht in der Klinik Buchinger Bodensee etwa 5\% der Patienten aus. Vor diesem Hintergrund ist ein additiver Effekt von einer medikamentösen Antikoagulation und Fasten zu beachten.

Ziel unserer Untersuchung war zu überprüfen, ob ein einwöchiges Fasten verglichen mit einer Kontrollgruppe (ovo-lacto-vegetarische Diät mit $1800 \mathrm{kcal}$ ) bereits bei gesunden, unbehandelten Probanden eine differente Wirkung auf die INR (International Normalized Ratio) zeigt. Die Auswahl gesunder Probanden wurde getroffen, um den Fasteneffekt ohne die Einflussfaktoren vorbestehender, das Gerinnungssystem beeinflussender Medikamente oder eines durch Adipositas veränderten Hormongleichgewichts beurteilen zu können.

Die Datensammlung erfolgte im Rahmen der «Summer School Naturheilverfahren» mit teilnehmenden Medizinstudenten, die Fasten bzw. ovo-lacto-vegetarische Diät als Selbsterfahrung durchführten. Die Teilnahme an den 
Messungen war freiwillig und die Gruppeneinteilung nicht randomisiert. Vor diesem Hintergrund konnte auf ein Ethikvotum verzichtet werden.

\section{Patienten und Methoden}

\section{Probanden}

Unsere Untersuchung wurde an einer bezüglich Alters- und Geschlechtsverteilung homogenen Gruppe von Medizinstudenten unter gleichen zeitlichen, räumlichen und klimatischen Bedingungen durchgeführt. Keiner der Teilnehmer litt an Essstörungen, und der jeweilige Body Mass Index (BMI) war mit Werten zwischen 18,5 und $25 \mathrm{~kg} / \mathrm{m}^{2}$ normal. Keiner der Probanden nahm Antikoagulanzien oder Thrombozytenaggregations hemmer; jeweils 5 Probandinnen in jeder Gruppe nahmen orale Antikontrazeptiva ein. Die Alters- und Geschlechterverteilung war in der Gruppe der Fastenden: 13 Probanden (9 weiblich, 4 männlich), Durchschnittsalter 25,2 Jahre (Standardabweichung (SD) 3 Jahre); in der Diätgruppe: 16 Probanden (14 weiblich, 2 männlich), Durchschnittsalter 24 (SD 1,9 Jahre). Alle Probanden wurden über die Untersuchung aufgeklärt und bestätigten ihr Einverständnis zur Teilnahme.

\section{Intervention}

Das Fasten wurde nach der Buchinger-Methode im Rahmen der Leitlinien [1] durchgeführt: Tag 0: «Entlastungstag» mit ca. 400 Gramm Obst; Tag 1: «Abführen» mit $40 \mathrm{~g}$ Natriumsulfat; Tage 1-4: Fasten (Saft oder Gemüsesuppe jeweils mittags und abends); Fastenbrechen an Tag 5; Tage 6 und 7: ovo-lacto-vegetarische Aufbaukost (ca. $1000 \mathrm{kcal} / \mathrm{Tag}$ ).

Die Teilnehmer der Diätgruppe nahmen über den Tag eine ovo-lacto-vegetarische Kost in 3 Mahlzeiten (morgens, mittags, abends) von insgesamt $1800 \mathrm{kcal} / \mathrm{Tag}$ zu sich.

\section{INR-Messung}

Die Messungen des INR-Wertes erfolgten in beiden Gruppen jeweils an den Tagen 1-7 zwischen 10 und 11 Uhr vormittags mit zwei Coagu-Check-XS ${ }^{\circledR}$ Geräten (Roche Diagnostics, Mannheim, Deutschland). Die Blutentnahmen wurden mittels Lanzette kapillar am Ohrläppchen nach vorheriger Desinfektion durchgeführt. Nach der Probengewinnung erfolgte eine Blutstillung mittels ca. einminütiger, manueller Kompression mit einem Tupfer.

\section{Statistik}

Die INR-Werte wurden in den Gruppen «Kontrolle» und «Fastende» zunächst mit einem nichtparametrischen ANOVA-Test (nach Brunner) ausgewertet. Dabei wurde untersucht,

- ob sich die INR-Werte zwischen den Gruppen überhaupt unterscheiden, - ob es eine Zeitabhängigkeit der Werte über die Beobachtungsdauer gibt.
Um bei einem Gruppenunterschied zu bewerten, ob die INR-Werte einer Gruppe höher oder niedriger als in der Vergleichsgruppe liegen, wurden die Daten mit einem nichtparametrischen Test unverbundener Stichproben nach Kruskal-Wallis untersucht. Als multipler Rangsummentest setzt er keine Normalverteilung voraus. Die genutzte Software war SPSS ${ }^{\circledR}$ (PASW Statistics - Version 17.0; SPSS, Chicago, Illinois, USA). Das Konfidenzintervall wurde mit $\mathrm{p}<0,05$ festgelegt.

\section{Ergebnisse}

Das Fasten wie auch die Diät wurden von allen Probanden problemlos vertragen. $\mathrm{Zu}$ gelegentlich geschilderten Nebenwirkungen wie Kopfschmerz oder Übelkeit nach Applikation des Natriumsulfates (Glaubersalz) kam es nicht. Die INR-Werte waren in den Gruppen signifikant unterschiedlich $(\mathrm{p}=0,02)$ und zeigten bei Fastenden sowie in der Diätgruppe signifikante Veränderungen im zeitlichen Verlauf $(\mathrm{p}=0,01)$. Die Tendenz der INR-Werte war unter Diät sinkend und unter Fastentherapie steigend. Dabei lagen die INR-Werte der Fastenden (Mittelwert INR $=1,04$; SD 0,082) signifikant $(\mathrm{p}=0,00011)$ über denen der Kontrollgruppe (Mittelwert INR =0,98; SD 0,078). Die Ergebnisse sind in Abbildung 1 dargestellt.

\section{Diskussion und Schlussfolgerungen}

Sowohl das Fasten als auch die ovo-lacto-vegetarische Diät nehmen Einfluss auf die plasmatische Gerinnung. Die Beobachtung des Abfallens der INR in der Diätgruppe deckt sich mit einer beschriebenen verkürzten Blutungszeit [11], wenn auch die Blutungszeit nicht allein durch die INR beeinflusst ist. Das Ausmass der nachgewiesenen Veränderungen der INR in beiden Gruppen ist bei gesunden Probanden klinisch nicht relevant und hat allenfalls Bedeutung, wenn z.B. chirurgische Interventionen bevorstehen [9]. So kann bei gesunden Probanden bei Fasten-
Abb. 1. Verlauf der INR-Werte (Mittelwert und Standardabweichung) bei Fastenden $(\mathrm{n}=13)$ und in der Diätgruppe $(\mathrm{n}=16)$ während des Interventionszeitraums. Am Tag 6 und 7 wurde bei den Fastenden ein Kostaufbau durchgeführt.

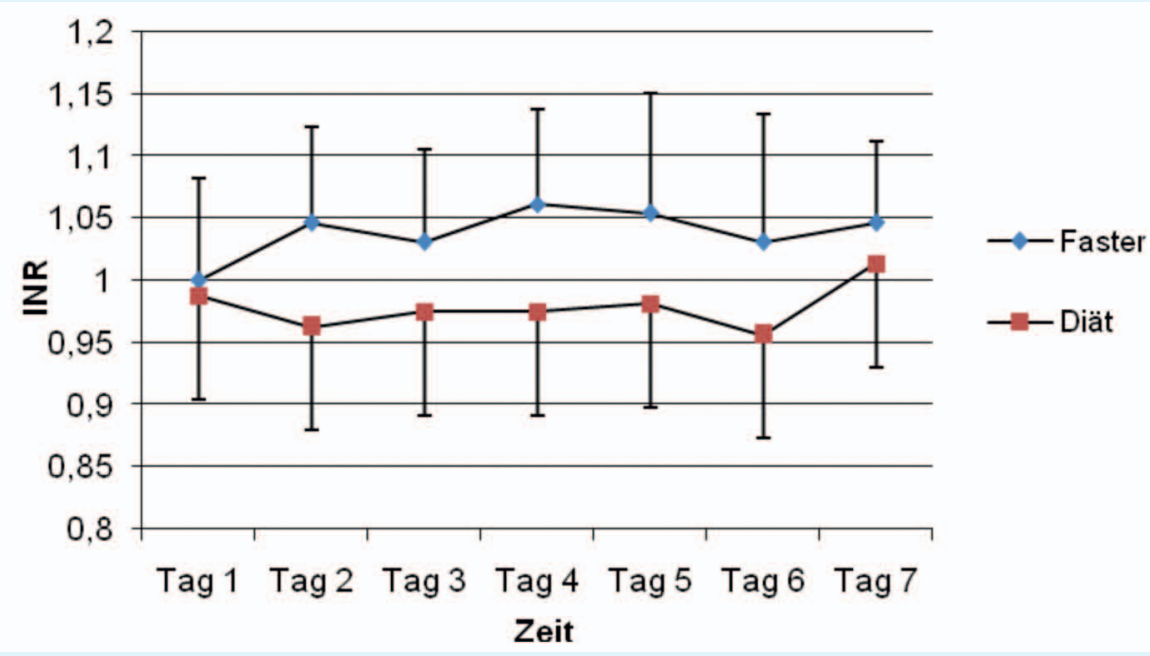

Drinda/de Toledo 
therapien oder ovo-lacto-vegetarischer Diät auf eine Kontrolle der Gerinnungswerte sicher verzichtet werden. Bei adipösen fastenden Patienten ist ein kumulativer Effekt denkbar, der sich einerseits durch Verminderung sonst thrombogen wirkender PAI-1-Spiegel und andererseits durch das Fasten ergibt. Die Dynamik wird verstärkt, wenn zusätzlich Gerinnungshemmer eingenommen werden. Bei mit Cumarinderivaten behandelten Patienten wird das Monitoring der Antikoagulation üblicherweise durch die INR-Messung realisiert. Da das Fasten die INR bereits bei unbehandelten, normalgewichtigen Personen stärker anhebt als eine ovo-lacto-vegetarische Diät, empfehlen wir zumindest bei mit Cumarinderivaten behandelten und fastenden Patienten eine engmaschigere INR-Kontrolle als sonst üblich. Die Messintervalle sollten während des Fastens zwischen täglich und alle 3 Tage betragen, da individuelle Reaktionen des Patienten und der (je nach Indikation unterschiedlichen) Ziel-INR beachtet werden müssen. Es ist grundsätzlich von einer Reduktion der Cumarinderivat-Dosis im Fasten auszugehen. Um die genaue Dynamik der Gerinnungswerte (linear oder nicht linear) von mit Cumarinderivaten sowie mit Heilfasten behandelten Patienten zu beurteilen, wären weitergehende Untersuchungen notwendig.

\section{Disclosure Statement}

Ein Interessenkonflikt der Autoren besteht nicht.

\section{Literatur}

1 Wilhelmi de Toledo F, Buchinger A, Burggrabe H, Gaisbauer M, Hölz G, Kronsteiner W, Kuhn C, Lischka E, Lischka N, Lützner H, May W, Melchart D, Michalsen A, Müller H, Peper E, Resch KL, RitzmannWidderich M, Wessel A, Wichert H, Stange R: Leitlinien zur Fastentherapie. Forsch Komplementärmed Klass Naturheilkd 2002;9:189-198.

2 Lützner H: Langzeitergebnisse aktiver Diätetik; Rehabilitationsstudie Baden. Therapeutikon 1988;9:512-519.

3 Peper E, Rogner J, Hettwer H: Stationäres Heilfasten. Z Allg Med 1997;73:146-150.

4 Müller H, Wilhelmi de Toledo F, Resch KL: Fasting followed by vegetarian diet in patients with rheumatoid arthritis: a systematic review. Scand J Rheumatol 2001;30:1-10.

5 Horne BD, May HT, Anderson JL, Kfoury AG, Bailey BM, McClure BS, Renlund DG, Lappé DL, Carlquist JF, Fisher PW, Pearson RR, Bair TL, Adams TD, Muhlestein JB; Intermountain Heart Collaborative Study: Usefulness of routine periodic fasting to lower risk of coronary artery disease in patients undergoing coronary angiography. Am J Cardiol 2008; 102:814-819.

6 Buchinger O: Das Heilfasten und seine Hilfsmethoden als biologischer Weg, ed 24. Stuttgart, Hippokrates, 2005, pp 14-15.

7 Huber R, Nauck M, Basler N, Haas B, Mattern M, Lüdtke R, Peter K: Effects of subtotal fasting on plasmatic coagulation, fibrinolytic status and platelet activation: a controlled pilot study in healthy subjects. Nutr Metab Cardiovasc Dis 2005;15:212-218.

8 Fahrner H: Fasten als Therapie. Stuttgart, Hippokrates, 1985, pp 37-39.

$\checkmark$ Usui Y, Tanimura H, Nishimura N, Kobayashi N, Okanoue T, Ozawa K: Vitamin $\mathrm{K}$ concentrations of the plasma and liver of surgical patients. Am J Clin Nutr 1990;51:846-852.

10 Lumeng CN: Adipose tissue macrophages: a piece of the PAI of metabolic syndrome. Sci Transl Med 2010;2:20.

11 Mezzano D, Muñoz X, Martínez C, Cuevas A, Panes O, Aranda E, Guasch V, Strobel P, Muñoz B, Rodríguez S, Pereira J, Leighton F: Vegetarians and cardiovascular risk factors: hemostasis, inflammatory markers and plasma homocysteine. Thromb Haemost 1999;81:913-917. 